\title{
Alpha-1 antitrypsin gene polymorphism in Chronic Obstructive Pulmonary Disease (COPD)
}

\author{
Sabri Denden ${ }^{1 *}$, Amel Haj Kheli1 ${ }^{1 *}$, Jalel Knani ${ }^{2}$, Ramzi Lakhdar ${ }^{1}$, Pascale Perrin ${ }^{3}$, \\ Gérard Lefranc ${ }^{4}$ and Jemni Ben Chibani ${ }^{1}$ \\ ${ }^{1}$ Biochemistry and Molecular Biology Laboratory, Faculty of Pharmacy, Monastir, Tunisia. \\ ${ }^{2}$ Pulmonology Department, CHU Tahar Sfar, Mahdia, Tunisia. \\ ${ }^{3}$ Institute of Evolution Sciences, University of Montpellier-II, France. \\ ${ }^{4}$ Institute of Human Genetics, University of Montpellier-II, France.
}

\begin{abstract}
Alpha-1-antitrypsin (AAT) plays an important role in the pathogenesis of emphysema, the pathological lesion underlying the majority of the manifestations of Chronic Obstructive Pulmonary Disease (COPD). In this study we tested the hypothesis that common AAT polymorphisms influence the risk of developing COPDs. We investigated PiM1 (Ala213Val), PiM2 (Arg101His), PiM3 (Glu376Asp), PiS (Glu264Val) and PiZ (Glu342Lys) SERPINA1 alleles in 100 COPD patients and 200 healthy controls. No significant differences were observed in allele frequencies between COPD patients and controls, neither did haplotype analysis show significant differences between the two groups. A cross-sectional study revealed no significant relationship between common SERPINA1 polymorphisms (PiM1, PiM2, PiM3) and the emphysematous type of COPD. In addition, $\mathrm{FEV}_{1}$ annual decline, determined during a two-year follow up period, revealed no difference among carriers of the tested polymorphisms.
\end{abstract}

Key words: alpha-1 antitrypsin, SERPINA1 polymorphisms, COPD, emphysema, lung function.

Received: January 16, 2009; Accepted: September 9, 2009.

Chronic obstructive pulmonary disease (COPD), a heterogeneous disorder, is a major cause of respiratory disability and the fourth major cause of death world-wide (World Health Organization, 2000). Exposure to cigarette smoke is recognized as the main environmental risk factor involved (Teramoto, 2007). Severe Alpha-1-antitrypsin deficiency (AATD) is a proven genetic risk factor, with about $80 \%$ of the subjects develop the disease between 30 to 40 years or earlier, in spite of only $1 \%-3 \%$ of COPD cases being due to severe AAT deficiency (Lomas and Silverman, 2001).

Alpha-1 antitrypsin (AAT) is a $52 \mathrm{kDa}$ protein synthesized primarily by hepatocytes. Its main function is to inhibit the activity of neutrophile elastase in the lung. This is a protease capable of destroying the major structural proteins of the alveolar wall. Plasma AAT deficiency results in accelerated elastin degradation, leading to a loss of ventilator function and the subsequent development of emphysema (Mahadeva and Lomas, 1998). The AAT coding gene SERPINA1 is highly polymorphic, with more than 125 SNPs reported in public databases, its most common alleles

Send correspondence to Denden Sabri. Biochemistry and Molecular Biology Laboratory, Faculty of Pharmacy, 1, AV. Avicenne 5019, Monastir, Tunisia. E-mail: denden_sabri@yahoo.fr.

*These authors contributed equally to this work. being the normal $\mathrm{M}$ alleles and its subtypes (PiM1Ala, PiM1Val, PiM2, PiM3), besides the deficient alleles PiS and PiZ (Crystal, 1990). About 95\% of the individuals with severe AATD are homozygous for PiZt (ATS/ERS, 2003), whereas PiSZ heterozygotes have approximately one-third of the normal AAT serum level, besides being highly prone to the development of diseases (Dahl et al., 2005). COPD risk among PiMZ heterozygotes has been previously analyzed with controversial results. Meta-analysis indicates a slight increase in risk of COPD in PiMZ individuals, with no significant lung-function impairment, compared to PiMM (Hersh et al., 2004).

A few studies on SERPINA1 common variants in COPD have been reported, also with controversial results. Matsuse et al. (1995), Shim (2001) and Kim et al. (2005) did not find a significant association between PiM1, PiM2 or PiM3 alleles and COPD, whereas Kwok et al. (2004) came across a significant increase in PiM1M3, PiM2M3 phenotypes and Gupta et al. (2005) reported a significant increase for the PiM3 allele in COPD patients.

For a better understanding of the association between SERPINA1 polymorphisms and COPD risk, we designed a case-control study to detect differences in the frequencies of common SNP alleles, haplotypes and genotypes, be- 
tween patients and controls in relation to common polymorphisms.

We also investigated the relationship between common SERPINA1 polymorphisms and main COPD clinical manifestations. The major AAT neutrophile elastase inhibitory role is observed in alveolar parenchyma, the subsequent deficiency in plasma AAT concentration mainly resulting in emphysema (Needham and Stockley, 2004). We therefore compared the distribution of SERPINA1 polymorphisms between the bronchial and emphysematous types of COPD. In addition, COPD patients underwent a two-year follow up, in order to evaluate the annual $\mathrm{FEV}_{1}$ (Forced Expiratory Volume in $1 \mathrm{~s}$ ) decline rate in relation to common SERPINA1 alleles. $\mathrm{FEV}_{1}$ is the hallmark of COPD since it is affected by inflammation and remodeling of the small airways as well as by emphysematous destruction of the terminal airspaces (Weiss et al., 2003).

The study population consisted of 100 COPD subjects who attended the Pneumology Department of Tahar Sfar Hospital in Mahdia. Inclusion criteria for patients with COPD were as follows: $\mathrm{FEV}_{1}<80 \%$ of predicted value adjusted for age, weight and height, and an improvement in $\mathrm{FEV}_{1}$ following bronchodilator inhalation $<12 \%$ of baseline $\mathrm{FEV}_{1}$. Asthmatic patients showing a persistent airflow obstruction were excluded. COPD phenotype identification was based on chest radiographic and high-resolution computerized tomography (HRCT) density findings. Clinical characteristics of COPD patients are summarized in Table S1. Follow-up examinations were conducted with patients over a two-year period after baseline, with annually repeated spirometry tests. AATD individuals were excluded from analysis. Two hundred healthy controls were enrolled for the case-control study. They were recruited from a blood donor's cohort of Fattouma Bourguiba Hospital in Monastir. Subjects with respiratory diseases, or any family history of lung disease, were excluded. The distribution of both patients and controls, according to demographic characteristics, is shown in Table S2. Prior written informed consent was obtained from all the subjects according to the research protocol approved by the local ethics committee.

Total genomic DNA was extracted from peripheral blood leucocytes by a phenol-chloroform method. PCRRFLP was employed for genotyping of PiM1 (Ala213 GㅁG $\rightarrow$ Val GTG), PiS (Glu264 GAA $\rightarrow$ Val GTA) and PiZ (Glu342 $\underline{\text { GAG }} \rightarrow$ Lys $\underline{\mathrm{A} A G)}$ polymorphisms, as previously described (Ferrarotti et al., 2004). Briefly, we performed PCR amplification using exon III primers to detect the $\mathrm{S}$ and 213Ala/Val variants and exon $\mathrm{V}$ for the $\mathrm{Z}$ variant. The reactions were carried out in an I-cycler Thermal Cycler (Bio-Rad Laboratories). $4 \mathrm{U}$ of SexAI and Hpy99I restriction enzymes (New England Biolabs) were used to digest $4 \mu \mathrm{L}$ each of exon III and exon $\mathrm{V}$ amplified DNA, respectively. Genotyping of PiM2 (Arg101 CGT $\rightarrow$ His CÁT) and PiM3 (Glu376 GAA $\rightarrow$ Asp GAC) was performed using hybridization probe analysis on Light Cycler
480 Roche apparatus (Roche Diagnostics), using a commercial real-time assay (LightMix ${ }^{\circledR}$, Roche Diagnostics). For each SNP, the primers flanking the SNP and the oligonucleotide probes were designed and synthesized by the manufacturer. The reaction mixture was prepared in a 96well PCR plate and processed according to manufacturer's instructions. Real-time PCR cycling conditions were as follows: $95{ }^{\circ} \mathrm{C}$ for $5 \mathrm{~min}$, followed by 35 cycles of $95{ }^{\circ} \mathrm{C}$ for $10 \mathrm{~s}, 62^{\circ} \mathrm{C}$ for $15 \mathrm{~s}$ and $72{ }^{\circ} \mathrm{C}$ for $15 \mathrm{~s}$. After amplification, PCR products were analyzed in a melting step of $40-95^{\circ} \mathrm{C}$. Melting data were analyzed using the Genescanning module of the LightCycler 480 software.

Annual FEV1 decline ( $\mathrm{ml} /$ year) was calculated as the difference between follow-up and baseline observed FEV1 values, divided by the number of months between the two surveys, and multiplied by 12 . SPSS v.10.0 software was used for statistical analysis. Categorical variables were presented as percentages, and intergroup differences were compared using $\chi^{2}$ test or Fisher's exact tests. Continuous variables, described as mean \pm standard deviation, were compared between the groups using Student's t test. Hardy Weinberg equilibrium tests and the estimation of allele and haplotype frequencies were performed using HPlus v. 2.5 software.

The frequency of PiM1, PiM2, PiM3, PiS and PiZ alleles and genotypes between COPD patients and healthy controls, was determined and compared (Table 1). Genotypes for all the polymorphisms were within HardyWeinberg proportions. There was no significant difference in the genotypic and allelic distribution of normal PiM1, PiM2 and PiM3 variants between subjects and controls. Deficient PiS and PiZ alleles were only reported in patients, with no apparent significant difference in relation to controls. Ten haplotypes were selected for studying by the expectation maximization procedure (Table 2), with no significant differences being detected between patients and controls by statistical comparison.

COPD patients were classified according to their predominant phenotype as follows: 53 subjects showed the bronchial type of the disease (chronic bronchitis group; mean age: $72.1 \pm 7.6$ years); 47 presented a predominant parenchymal destructive change (centrolobular and panlobular emphysema groups; mean age: $69.5 \pm 12.2$ years). Univariate analysis was employed to verify whether there were differences in COPD phenotypes among SERPINA1 genotypes (M1Ala containing $v s$. non M1Ala containing; M2 containing vs. non M2 containing and M3 containing $v s$. non $\mathrm{M} 3$ containing). The relationship between $\mathrm{FEV}_{1}$ annual decline, smocking and BMI and COPD phenotypes was also examined. No significant differences were detected (Table 3).

Lung function impairment in patients was assessed by the annual $\mathrm{FEV}_{1}$ decline rate. After exclusion of AAT deficient individuals, 96 patients underwent a two-year follow up Annual $\mathrm{FEV}_{1}$ decline means were compared according 
Table 1 - SERPINAlgenotypes and alleles in COPD patients and controls.

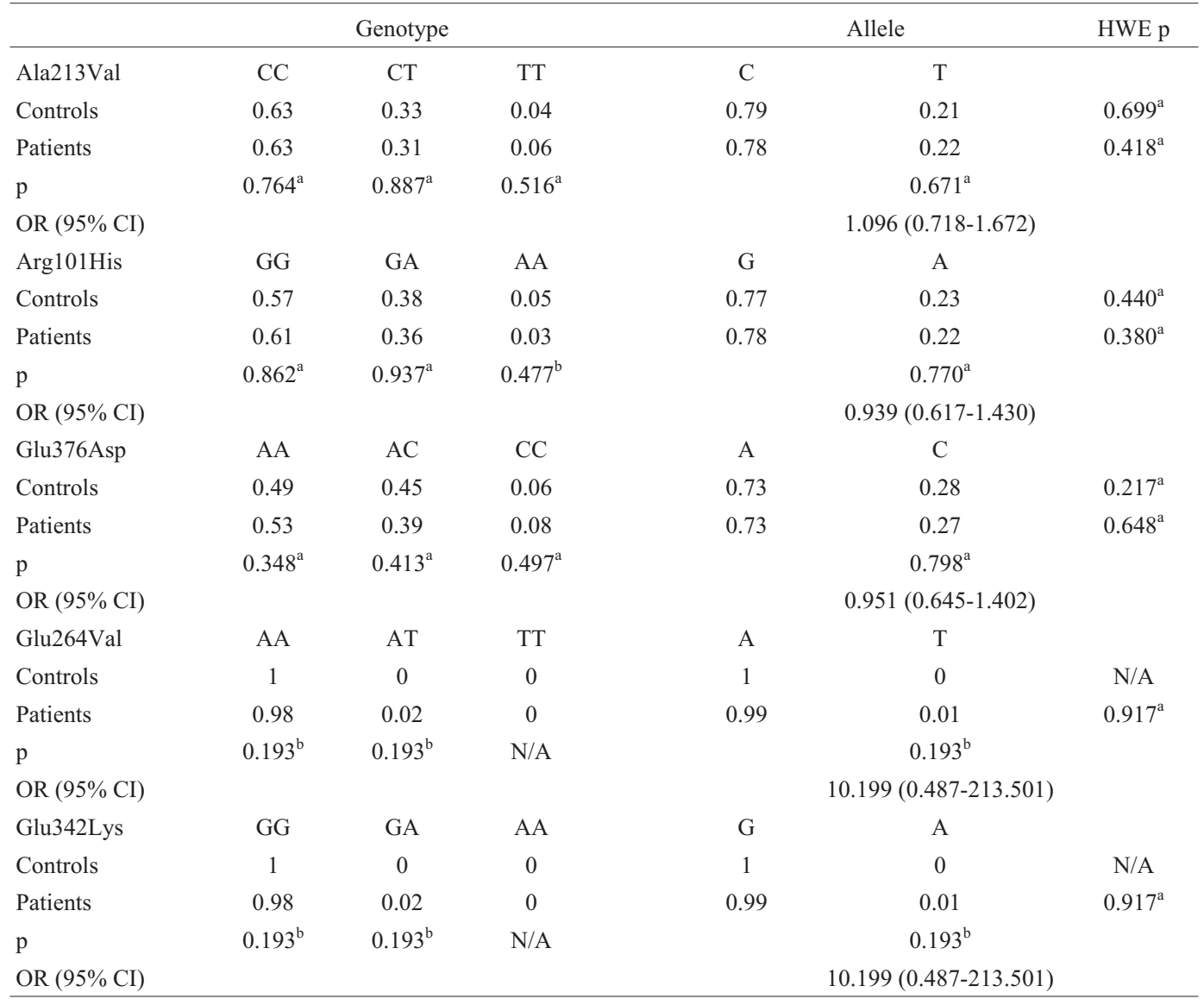

HWE: Hardy-Weinberg Equilibrium. ${ }^{a}$ Pearson's $\chi^{2}$ test; ${ }^{b}$ Fisher's exact test.

Table 2 - SERPINA1 SNP haplotypes in COPD patients and controls.

\begin{tabular}{lccccc}
\hline Haplotype $^{\mathrm{a}}$ & Patients & Controls & $\mathrm{p}^{\mathrm{b}}$ & OR & $95 \%$ CI \\
\hline GTAGA & 0.48265 & 0.48773 & 1 & 1 & N/A \\
GCAGA & 0.21581 & 0.20572 & 0.864 & 1.04 & $0.67-1.61$ \\
ATAGC & 0.18798 & 0.20385 & 0.683 & 0.91 & $0.56-1.46$ \\
GTAGC & 0.08046 & 0.07999 & 0.974 & 0.99 & $0.50-1.96$ \\
ATAGA & 0.01730 & 0.02270 & 0.663 & 0.77 & $0.24-2.50$ \\
ATTGA & 0.00526 & 0.00000 & N/A & N/A & N/A \\
ATAAC & 0.00524 & 0.00000 & N/A & N/A & N/A \\
GCAAA & 0.00524 & 0.00000 & N/A & N/A & N/A \\
GTAAA & 0.00005 & 0.00000 & N/A & N/A & N/A \\
ACAGC & 0.00001 & 0.00000 & N/A & N/A & N/A \\
\hline
\end{tabular}

${ }^{a}$ Haplotype frequency determined using expectation maximization method; ${ }^{b}$ Fisher's exact test.

to age, smoking habits and SERPINA1 polymorphisms. No significant relationship between annual $\mathrm{FEV}_{1}$ decline and the tested variables was detected (Table 4).

In summary, our findings are consistent with observations that there is no significant difference in the frequency of common SERPINA1 variants in COPD patients, when
Table 3 - Association studies of bronchial and emphysematous COPD types with annual FEV1 decline $\left(\triangle \mathrm{FEV}_{1}\right)$, cigarette smoking, body mass index (BMI) and SERPINA1 genotypes.

\begin{tabular}{lccc}
\hline Type/Class & \multicolumn{2}{c}{ COPD phenotype } & \multirow{2}{*}{$\mathrm{p}$} \\
\cline { 2 - 3 } & \multicolumn{1}{c}{ CB } & CLE+PEL & \\
\hline$\Delta$ FEV $_{1}$ (ml/year) & $171 \pm 137$ & $232 \pm 239$ & $0.243^{\mathrm{a}}$ \\
Cumulative cigarette & $59.41 \pm 29.22$ & $51.97 \pm 28.68$ & $0.230^{\mathrm{a}}$ \\
consumption & & & \\
BMI (kg/m $\left.{ }^{2}\right)$ & $24.33 \pm 3.70$ & $22.77 \pm 5.21$ & $0.117^{\mathrm{a}}$ \\
M1Ala & 0.41 & 0.53 & \\
No M1Ala & 0.58 & 0.47 & $0.306^{\mathrm{b}}$ \\
M2 & 0.45 & 0.45 & \\
No M2 & 0.54 & 0.54 & \multirow{2}{*}{$1^{\mathrm{b}}$} \\
M3 & 0.39 & 0.53 & \\
No M3 & 0.60 & 0.46 & $0.184^{\mathrm{b}}$ \\
\hline
\end{tabular}

CB: Chronic Bronchitis; CLE: Centrolobular Emphysema; PLE: Panlobular Emphysema; Cumulative cigarette consumption = number of packs smoked per day multiplied by years of consumption. M1Ala: heterozygous and homozygous for Ala213 allele; no M1Ala: homozygous for Val213 allele; M2: heterozygous and homozygous for His101allele; no M2: homozygous for Arg101 allele; M3: heterozygous and homozygous for Asp376 allele; no M3: homozygous for Glu376 allele.

${ }^{\text {a }}$ Student's t test; ${ }^{\text {bPearson's }} \chi^{2}$ test. 
Table 4 - Association studies of lung function impairment with smoking, body mass index (BMI), age and SERPINA1 genotypes.

\begin{tabular}{lcc}
\hline Type/class & $\Delta \mathrm{FEV}_{1}(\mathrm{~mL} /$ year $)$ & $\mathrm{p}^{\mathrm{a}}$ \\
\hline $\begin{array}{l}\text { Smoking status } \\
\quad \text { Never }\end{array}$ & $151 \pm 131$ & \\
$\quad$ Smoker & $197 \pm 191$ & 0.683 \\
$\quad$ Cumulative cigarette consumption & & \\
$\quad<$ mean & $164 \pm 179$ & \\
$\quad>$ mean & $230 \pm 194$ & 0.197 \\
BMI (kg/m $\left.{ }^{2}\right)$ & & \\
$\quad<$ mean BMI & $221 \pm 239$ & \\
$\quad>$ mean BMI & $178 \pm 144$ & 0.408 \\
Age & & \\
$\quad<$ mean age & $165 \pm 109$ & \\
$\quad>$ mean age & $207 \pm 211$ & 0.455 \\
M1Ala & $195 \pm 177$ & \\
No M1Ala & $194 \pm 205$ & $0.983^{\mathrm{a}}$ \\
M2 & $195 \pm 156$ & \\
No M2 & $195 \pm 204$ & 0.999 \\
M3 & $205 \pm 212$ & \\
No M3 & $187 \pm 152$ & 0.726 \\
\hline
\end{tabular}

Cumulative cigarette consumption $=$ number of packs smoked per day multiplied by years of consumption. M1Ala: heterozygous and homozygous for Ala213 allele; no M1Ala: homozygous for Val213 allele; M2: heterozygous and homozygous for His101 allele; no M2: homozygous for Arg101 allele; M3: heterozygous and homozygous for Asp376 allele; no M3: homozygous for Glu376 allele.

${ }^{a}$ Student's $t$ test.

compared to healthy controls. We also verified that there is no correlation between these alleles and the manifestation of emphysema, since there was no difference in their distribution in patients with bronchial and emphysematous types of COPD. Furthermore, no significant relationship between SERPINA1 polymorphisms and annual $\mathrm{FEV}_{1}$ decline evaluated over a two-year period in COPD patients was found. To our knowledge, this is the first report on clinical manifestations of COPD in relation to common AAT variants.

\section{Acknowledgments}

This work was supported by a grant from the Ministry of Education and Scientific Research in Tunisia.

\section{References}

ATS/ERS Statement (2003) Standards for the diagnosis and management of individuals with alpha-1 Antitrypsin deficiency. Lung disease section. Am J Respir Crit Care Med 168:823849.

Crystal RG (1990) $\alpha 1$-antitrypsin deficiency, emphysema, and liver disease genetic basis and strategies for therapy. J Clin Invest 85:1343-1352.

Dahl M, Hersh CP, Ly NP, Berkey CS, Silverman EK and Nordestgaard BG (2005) The protease inhibitor PI*S allele and COPD: A meta-analysis. Eur Respir J 26:67-76.
Ferrarotti I, Zorzetto M, Scabini R, Mazzola P, Campo I and Luisetti M (2004) A novel method for rapid genotypic identification of alpha1-antitrypsin variants. Diagn Mol Pathol 12:160-163.

Gupta J, Bhadoria DP, Lal MK, Kukreti R, Chattopadhaya D, Gupta VK, Dabur R, Yadav V, Chhillar AK and Sharma GL (2005) Association of the PIM3 allele of the alpha-1-antitrypsin gene with chronic obstructive pulmonary disease. Clin Biochem 38:489-491.

Hersh CP, Dahl M, Ly NP, Berkey CS, Nordestgaard BG and Silverman EK (2004) Chronic obstructive pulmonary disease in alpha 1-antitrypsin PI MZ heterozygotes: A metaanalysis. Thorax 59:843-849.

Kim CH, Yim JJ, You CG, Lee CT, Kim YW, Han SK and Shim YS (2005) Alpha-antitrypsin genotypes in Korean patients with chronic obstructive pulmonary disease. Respirology 10:223-228.

Kwok JSY, Lawton JWM, Yew WW, Chau CH, Lee J and Wong PC (2004). Protease inhibitor phenotypes and serum alpha-1-antitrypsin levels in patients with COPD: A study from Hong Kong. Respirology 9:265-270.

Lomas DA and Silverman EK (2001) The genetics of chronic obstructive pulmonary disease. Respir Res 2:20-26.

Mahadeva R and Lomas DA (1998) Genetics and respiratory disease* 2: Alpha 1 antitrypsin deficiency, cirrhosis and emphysema. Thorax 53:501-505.

Matsuse T, Fukuchi Y, Matsui H, Sudo E, Nagase T and Orimo H (1995) Effect of cigarette smoking on pulmonary function in each phenotype $\mathrm{M}$ of a-1-Protease inhibitor*. Chest 107:395-400.

Needham M and Stockley RA (2004) $\alpha 1$-antitrypsin deficiency * 3: Clinical manifestations and natural history. Thorax 59:441-445

Shim YS (2001) Epidemiological survey of chronic obstructive pulmonary disease and alpha-1 antitrypsin deficiency in Korea. Respirology 6:S9-S11.

Teramoto S (2007) 1. COPD pathogenesis from the viewpoint of risk factors. Intern Med 46:77-79.

Weiss ST, DeMeo DL and Postma DS (2003) COPD: Problems in diagnosis and measurement. Eur Respir J 21:4s-12s.

\section{Internet Resources}

HPlus software v 2.5, http://qge.fhcrc.org/hplus (June 18, 2007).

World Health Organization (2000) the World Health Report 2000, http://www.who.int/whr/2000/en/ (December 3, 2008).

\section{Supplementary Material}

The following online material is available for this article: - Table S1 - Clinical characteristics of COPD patients at baseline.

- Table S2 - Demographic characteristics of COPD patients and healthy controls

This material is made available as part of the on-line article from http://www.scielo.br.gmb.

\section{Associate Editor: Francisco Mauro Salzano}

License information: This is an open-access article distributed under the terms of the Creative Commons Attribution License, which permits unrestricted use, distribution, and reproduction in any medium, provided the original work is properly cited. 
Table S1 - Clinical characteristics of COPD patients at baseline.

\begin{tabular}{|c|c|}
\hline Clinical characteristics & COPD Patients $(\mathrm{n}=100)$ \\
\hline \multicolumn{2}{|l|}{ COPD phenotype } \\
\hline $\mathrm{CB}$ & $52 \%$ \\
\hline $\mathrm{CB}+\mathrm{CLE}$ & $35 \%$ \\
\hline PLE & $13 \%$ \\
\hline \multicolumn{2}{|l|}{ Spirometry } \\
\hline $\mathrm{FEV}_{1}$ & $1.16 \pm 0.61$ \\
\hline $\mathrm{FEV}_{1} \%$ pred & $41.7 \pm 16.8$ \\
\hline $\mathrm{FVC}$ & $1.55 \pm 0.78$ \\
\hline FVC $\%$ pred & $43.7 \pm 18.5$ \\
\hline $\mathrm{RV} \%$ & $2.7 \pm 3.4$ \\
\hline \multicolumn{2}{|l|}{ Blood gases } \\
\hline $\mathrm{PaO}_{2}$ & $69.2 \pm 10.6$ \\
\hline $\mathrm{PaCO}_{2}$ & $43.2 \pm 10$ \\
\hline $\mathrm{SaO}_{2}$ & $93 \pm 3.8$ \\
\hline \multicolumn{2}{|l|}{ COPD stage } \\
\hline I & $2 \%$ \\
\hline II & $24 \%$ \\
\hline III & $48 \%$ \\
\hline IV & $26 \%$ \\
\hline
\end{tabular}

CB: Chronic Bronchitis; CLE: Centrolobular Emphysema; PLE: Panlobular Emphysema; $\mathrm{FEV}_{1}$ : Forced Expiratory Volume in $1 \mathrm{~s}(\mathrm{~L})$; FVC: Forced Vital Capacity (L); $\mathrm{FEV}_{1} \%$ pred: percentage of the predicted $\mathrm{FEV}_{1}$ value adjusted to age, height and weight. $\mathrm{FVC} \%$ pred: percentage of the forecast FVC value adjusted to age, height and weight. RV: Reversibility of baseline $\mathrm{FEV}_{1}$ following bronchodilator inhalation; $\mathrm{PaO} 2$ : arterial oxygen partial pressure $(\mathrm{mm} \mathrm{Hg}) ; \mathrm{PaCO}_{2}$ : arterial carbon monoxide partial pressure (mm $\mathrm{Hg}) ; \mathrm{SaO}_{2}$ : Arterial oxygen saturation (\%).

Table S2 - Demographic characteristics of COPD patients and healthy controls.

\begin{tabular}{lccc}
\hline Demographic characteristics & COPD patient $(\mathrm{n}=100)$ & Controls $(\mathrm{n}=200)$ & $\mathrm{p}$ \\
\hline Age & $69.45 \pm 12.34$ & $43.86 \pm 8.97$ & $0.00^{\mathrm{a}}$ \\
male:female & $98: 2$ & $189: 11$ & $0.15^{\mathrm{b}}$ \\
BMI & $24.5 \pm 4.0$ & $24.4 \pm 2.9$ & $0.80^{\mathrm{a}}$ \\
Smoking status & & & \\
$\quad$ Never & 7 & 16 & \\
Ex & 52 & 89 & $0.09^{\mathrm{b}}$ \\
Current & 41 & 95 & $0.14^{\mathrm{a}}$ \\
Cumulative cigarette consumption & $53.74 \pm 29.76$ & $48.41 \pm 23.86$ & \\
Geographic origin in Tunisia & & & \\
North & 0 & 4 & $0.08^{\mathrm{b}}$ \\
Center & 100 & 4 & \\
South & 0 & & \\
\hline
\end{tabular}

BMI: Body mass index, weight $(\mathrm{kg}) /(\text { height }(\mathrm{m}))^{2}$. Cumulative cigarette consumption = number of packs smoked per day multiplied by years of consumption.

${ }^{\mathrm{a}}$ Student's t test; ${ }^{\mathrm{b}}$ Pearson's $\chi^{2}$ test. 